\title{
Method of assessment of the transport system functioning with the application of the simulation model of the operating process
}

\author{
Klaudiusz Migawa ${ }^{1, *}$, Agnieszka Sołtysiak ${ }^{1}$ and Magda Czyżewska ${ }^{1}$ \\ ${ }^{1}$ University of Science and Technology, Faculty of Mechanical Engineering, 85-796 Bydgoszcz, \\ Poland
}

\begin{abstract}
In the article, there is presented the description of the algorithm of the operating process of the simulation model characteristics' determination realized in the transport system. The simulation model of the means of transport's operating process has been developed based on the that process's mathematical model (semi-Markov model). Due of the assumed principles, the constructed model can be applied for the purposes of assessment and control of the operating process because of criterion of different types. Analysis of performance's assessment of the operating means of transport is one of the ways of the possibilities of correct realization of the assigned transportation tasks in the transport systems. The simulation model of the operating process presented in the work, makes it possible to assess performance of the transport system's operation from the point of view of the selected assessment criteria: availability of the means of transport and the unit income generated in the process's modelling conditions. Based on the results of the tests conducted in the real system of the means of transport operating system, the model's input data have been developed and simulation experiments have been conducted. As an effect, there have been determined the exemplary values of the analyzed characteristics of the transport system's operation performance analyses.
\end{abstract}

\section{Introduction}

One of the ways making it possible to assess effectively and to control the quality of the complex technical objects in operating systems, is application of the mathematical and simulation models for describing and analysis purposes of the operating processes performed in these systems. Models of that type, may be applied for assessment and the quality's control of the complex technical processes from the point of view of different assessment criteria, such as costs, incomes, reliability, availability, safety, productivity [7, 11]. The issues concerning the operating process's steering performed in the complex technical systems depending on the selected assessment criterion, has been presented in many studies $[1,2,3,9,12]$. Depending on the type of selected research problems, appropriate mathematical models and methods of determining the optimum or quasioptimum solutions are applied. Due to a considerable complexity of the processes realized in real systems of the technical objects operating, there occurs the necessity of applying appropriate methods and tools, including the stochastic models $[1,4,5]$, and computer simulation programmes, ensuring effective performance of the researches with reference to the models of the considered operating processes, and analysis of the obtained results $[6,8$, $10,13,14]$. These models ensure the possibility of assessing and steering the operating performance of the complex technical systems from the point of view of selected criteria.

* Corresponding author: klaudiusz.migawa@utp.edu.pl 
In the study there are presented the results of operating performance of a selected, real system of technical objects operating, obtained based on the researches of the developed, simulation operating process realized in the considered system. The operating process's simulation model presented in the study, makes it possible to assess performance of the transport system's operation on account of two assessment criteria: availability of the means of transport and the unit income generated as a result of the operating process's realization. The simulation model applied in the study, has been developed in order to ensure a quicker and easier analysis and possibility to consider different variants of the modelled operating processes (for ex. by the change of the values of the modelled process's parameters or the number of considered technical objects). The constructed operating process's simulation model has been developed based on the that process's mathematical model (semi-Markov model). The mathematical operating process' model, has been constructed based on the analysis of the operating states and events' spaces (operating process's occurrence model) concerning the technical objects (means of transport) operating in the analyzed real system.

\section{Model of the means of transport operating process}

\subsection{Occurrence model of the operating process}

Below there is presented the occurrence model of the analyzed means of transport's operating process. As a result of the identification of the examined means of transport's operating process, there have been determined the essential operating states of that process, and possible transitions between the singled-out states. On that basis, the graph of the operating processes states' changes presented in figure 1, has been constructed. The following operating states of the technical object have been singled out in the analyzed model: 1 - stoppage at the depot yard, 2 - carrying out of transportation task, 3 - stoppage at route caused by damage, 4 - stoppage caused by a collision, 5 - intervention following the collision, 6 - repair following damage or collision, 7 - preventive diagnosis, 8 preventive repair, 9 - supply, 10 - servicing (on the day of use, periodical, seasonal).

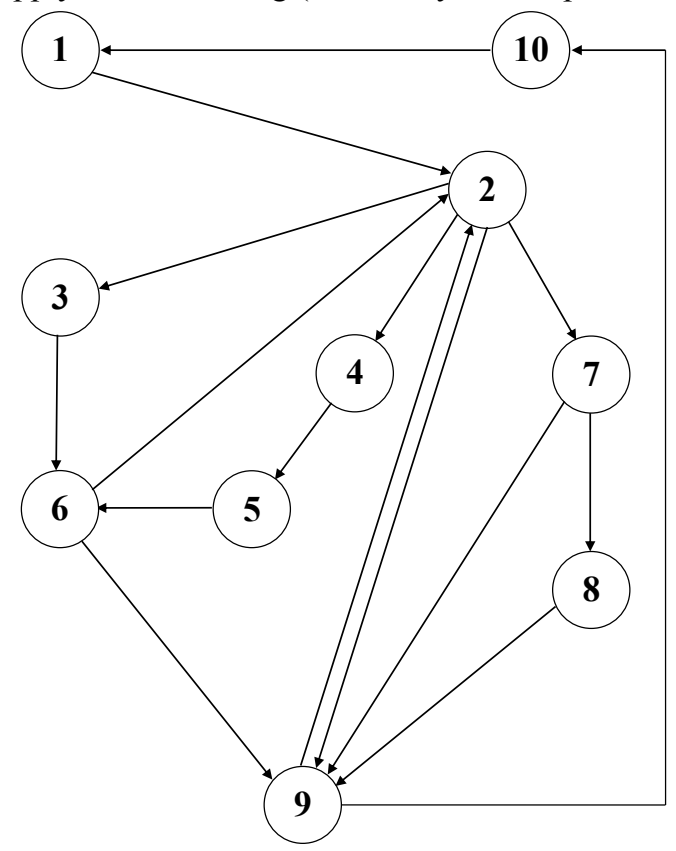

Fig. 1. Directed graph of the means of transport operating process's representing. 


\subsection{Mathematical model of the operating process}

In the study, the mathematical model of the means of transport's operating process, has been built assuming, that the model of that process there is the uniform semi-Markov $X(t)$ process. The semi-Markov process $X(t)$ is the process, in which the times between the changes of subsequent process's states have arbitrary probability distributions and transition to the next state depends only on the current process's state. Applying the semiMarkov processes to mathematical operating processes' modeling, the following assumptions have been applied:

- modelled process has a definite number of states $i=1,2, \ldots, 10$,

- if a technical object at the moment $t$ is in the state $i$, then $X(t)=i$,

- random process $X(t)$, being the mathematical model of the operating process, is the uniform process,

- at the time $t=0$, the process is in the state 1 (state 1 is the initial state), that means $P\{X(0)=1\}=1$.

The uniform semi-Markov process is determined unequivocally when there has been given the process's initial distribution and its kernel. From the accepted assumptions and based on the directed graph presented in figure 1, the initial distribution has the form of

$$
p_{i}(0)=\left\{\begin{array}{ll}
1, & g d y i=1 \\
0, & g d y i \neq 1
\end{array}, i=1,2, \ldots 10\right.
$$

where

$$
p_{i}(0)=P\{X(0)=i\}, \quad i=1,2, \ldots 10
$$

while the process's kernel $Q(t)$ has the form of

$$
Q(t)=\left|\begin{array}{cccccccccc}
0 & Q_{1,2}(t) & 0 & 0 & 0 & 0 & 0 & 0 & 0 & 0 \\
0 & 0 & Q_{2,3}(t) & Q_{2,4}(t) & 0 & 0 & Q_{2,7}(t) & 0 & Q_{2,9}(t) & 0 \\
0 & 0 & 0 & 0 & 0 & Q_{3,6}(t) & 0 & 0 & 0 & 0 \\
0 & 0 & 0 & 0 & Q_{4,5}(t) & 0 & 0 & 0 & 0 & 0 \\
0 & 0 & 0 & 0 & 0 & Q_{5,6}(t) & 0 & 0 & 0 & 0 \\
0 & Q_{6,2}(t) & 0 & 0 & 0 & 0 & 0 & 0 & Q_{6,9}(t) & 0 \\
0 & 0 & 0 & 0 & 0 & 0 & 0 & Q_{7,8}(t) & Q_{7,9}(t) & 0 \\
0 & 0 & 0 & 0 & 0 & 0 & 0 & 0 & Q_{8,9}(t) & 0 \\
0 & Q_{9,2}(t) & 0 & 0 & 0 & 0 & 0 & 0 & 0 & Q_{9,10}(t) \\
Q_{10,1}(t) & 0 & 0 & 0 & 0 & 0 & 0 & 0 & 0 & 0
\end{array}\right|
$$

where:

$$
\begin{gathered}
Q_{i, j}(t)=P\left\{X\left(\tau_{n+1}\right)=j, \tau_{n+1}-\tau_{n} \leq t \mid X\left(\tau_{n}\right)=i\right\} \\
Q_{i, j}(t)=p_{i j} \cdot F_{i j}(t) \\
p_{i j}(t)=P\{X(t)=j \mid X(0)=i\}
\end{gathered}
$$

is the conditional probability of transition from the state $i$ to the state $j$

$$
F_{i j}(t)=P\left\{\tau_{n+1}-\tau_{n} \leq t \mid X\left(\tau_{n}\right)=i, X\left(\tau_{n+1}\right)=j\right\}
$$

is the distribution function of a random variable determining the duration time of the state $i$, on condition, that the next state there shall be state $j$.

The limiting probabilities $p_{i}{ }^{*}$ of staying in the states of the considered process $X(t)$, have been determined based on the limiting theorem for the semi-Markov processes [1]

$$
p_{i}^{*}=\frac{\pi_{i} \cdot \bar{\Theta}_{i}}{\sum_{i \in S} \pi_{i} \cdot \bar{\Theta}_{i}}
$$

where: 
$\bar{\Theta}_{i}$ - average unconditional duration times of the states of the process $X(t)$,

$\pi_{i}$ - probability of stationary distribution of the inserted Markov chain, which meets the system of linear equations

$$
\sum_{i \in S} \pi_{i} \cdot p_{i j}=\pi_{j}, \quad j \in S, \quad \sum_{i \in S} \pi_{i}=1
$$

where

$p_{i j}$ - conditional probabilities of transition from state $i$ to state $j$, according to the dependence

$$
p_{i j}=\lim _{t \rightarrow \infty} p_{i j}(t)
$$

Considering the above discussions, the following describing formulas have been determined:

- value of the technical object availability

$$
A=\sum_{i \in S_{A}} p_{i}^{*}=\frac{\sum_{i \in S_{A}} \pi_{i} \cdot \bar{\Theta}_{i}}{\sum_{i \in S} \pi_{i} \cdot \bar{\Theta}_{i}}
$$

where

$S_{A} \subset S=\{1,2, \ldots, 10\}-$ set of availability states of the semi-Markov process model $X(t)$,

- value of the average unit income

$$
C=\sum_{i \in S} p_{i}^{*} \cdot c_{i}=\frac{\sum_{i \in S} \pi_{i} \cdot \bar{\Theta}_{i} \cdot c_{i}}{\sum_{i \in S} \pi_{i} \cdot \bar{\Theta}_{i}}
$$

where

$c_{i}$ - unit costs generated in the states of the process $X(t)$.

\section{Simulation model of the performance assessment}

The developed simulation model of the operating process, makes it possible to asses performance of the transport system's operation on acount of two assessment criteria: availability of the means of transport and the unit income generated as a result of the operating process's realization. The simulation programme, constituting the computer realization of the model of the means of transport operating model, has been constructed in just the same manner, making it possible to realize the simulation experiments with the use of the input data sets prepared based on operating data obtained from the researches concerning the real system of the means of transport's operating. The developed model of the operating simulation's programme, allows conducting simulation experiments for different numbers of operating occurrences (changes of the process's states), simulation time intervals, both in case of a single technical object as well as of a group of technical objects. In the simulation programme, subsequent realizations of the duration times of the operating process's model states, are determined with the use of the pseudorandom numbers' generating algorithms, generating the values from exponential, gamma, normal, log-normal and Weibull distributions. The structure of the simulation programme has been developed in such a way that to make it possible for the simulation experiment to represent in the course of realization the real set of technical objects (means of transport), and succession of occurrences finding fulfillment in the studies on the real transportation system, concerning individual technical objects. The developed simulation programme of the operating process' model was written by: R Core Team (2018). R: A language and environment for statistical computing. R Foundation for Statistical Computing, Vienna, Austria. URL http://www.R-project.org/. In figure 2, there is presented the block diagram 
of operation of the process of the technical objects operating simulation model's programme.

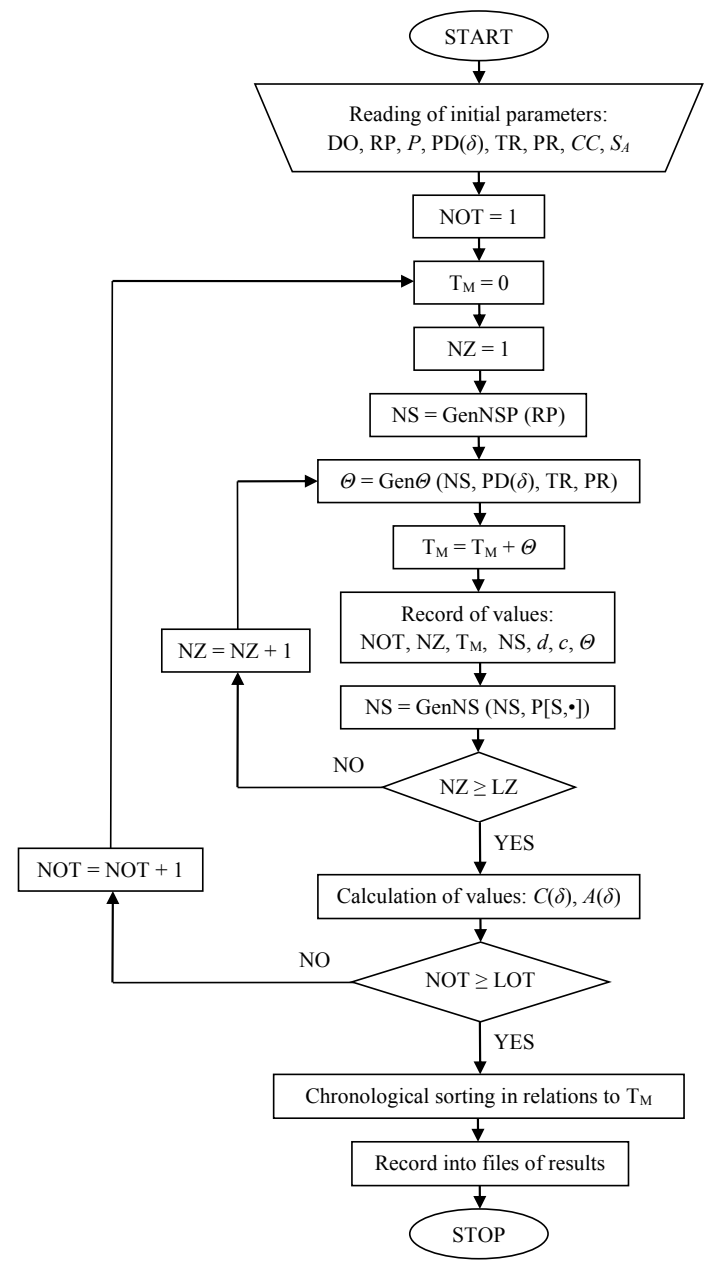

Fig. 2. Block diagram of the operation of the process of the technical objects operating simulation model's programme.

For the purposes of the simulation experiment's realization, it is necessary to prepare the set of the determined input data:

a) DO (general data characterizing the simulation experiment):

NOT - number of technical object in the simulation experiment,

$\mathrm{NZ}$ - number of occurrences for a technical object in the simulation experiment,

$m$ - number of states of the process, determined on the basis of the set of states of the modelled operating process $S=\{1,2, \ldots, m\}$

ND - number of decisions possible to be applied in individual states of the modelled operating process,

$\mathrm{N} \delta$ - number of strategies $\delta$ possible to be applied in the simulation experiment;

The strategy $\delta$ shall mean the sequence, the words of which are vectors, made of decisions $d_{i}^{(k)}\left(t_{n}\right)$ taken in individual states of the process $X(t)$, in subsequent moments $t_{n}$ of these states' changes 


$$
\delta=\left\{\left[d_{1}^{(k)}\left(t_{n}\right), d_{2}^{(k)}\left(t_{n}\right), \ldots, d_{m}^{(k)}\left(t_{n}\right)\right]: \quad n=0,1,2, \ldots\right\}
$$

In case when decisions taken in subsequent states of the process shall not depend on the moment $t_{n}$, in which they are taken, that is $d_{i}^{(k)}\left(t_{n}\right)=d_{i}^{(k)}$, then the strategy $\delta$ is called the stationary strategy. Then, the formula (13) shall assume the form

$$
\delta=\left[d_{1}^{(k)}, d_{2}^{(k)}, \ldots, d_{m}^{(k)}\right]
$$

b) RP (process initial distribution):

stochastic vector of the number of constituents equal to the number of the states of modelled operating process, determining distribution of staying in the states of the modelled operating process at the beginning of the simulation experiment;

c) $P$ (probability matrix of the process's states changes):

square matrix of the degree equal to the number of the states of the modelled operating process, in which each line is the stochastic vector determining distribution of the process's states changes, on condition that the current state of the process shall be determined by that line's number;

d) PD (probability matrix of the decision's selection in the states of the process):

rectangular matrix of the number of lines equal to the number of states of the modelled operating process and the number of columns equal to the number of decisions possible to be taken in each state of the modelled process, composed of stochastic lines determining probability distributions of a given decision's selection at the moment of a technical objects entering a given state of a modelled process;

In case of applying of a deterministic strategy $\delta$, values of the matrix $\operatorname{PD}(\delta)$ elements determine the stationary vector, the components of which are the numerical codes defining the deterministic decisions applied in individual states of the modelled operating process;

e) TR, PR (types and parameters of distributions items of remaining in the process states): the developed programme includes the functions generating the values from the exponential, gamma, normal, log-normal and Weibull distributions on preset parameters;

f) $C C$ (unit incomes matrix in the process states):

rectangular matrix of the number of lines equal to the number of states of the modelled operating process, and the number of columns equal to the number of decisions possible to be taken in each state of the modelled process, in which, for each state of the process and for each decision possible to be taken in a given state (at the meeting point of a line and a column), there is determined the unit income's value (per time unit) generated, when a technical object stays in a given state (determined by a line's number), while taking a decision (determined with the column's number);

g) $S_{A}$ (set of the process's availability states):

set $S_{A}$ of the numerical codes ascribed to the availability states of the operating process's model, determined based on the set $S$ of numerical codes ascribed to the operating process's model states $\left(S_{A} \subset S=\{1,2, \ldots, m\}\right)$.

Additionally, in figure 2, there have been used the following symbols: GenNSP generating of the model's initial state number, GenNS - generating of the model's current state number, Gen $\Theta$ - generating of the value of time of staying in the current model's state.

In the course of the simulation experiment's conducting, at each moment in which change of the modelled operating process occurs (for a considered technical object), the following data are recorded in the results' file:

- number of the technical object NOT, 
- number of the current occurrence NZ,

- moment of the current occurrence (current time of the model $\mathrm{T}_{\mathrm{M}}$ ),

- number of the current state of the model NS,

- current decision $d$,

- value of the unit income c concerning remaining of the technical object in the current state,

- generated value of the time of remaining in the current state $\Theta$.

Then, for the data set generated in the course of the simulation experiment's conducting, the values of characteristics of the transport system's operation performance assessment (for the applied strategy $\delta$ ) are determined:

- result determining the value of the technical objects availability

$$
A(\delta)=\frac{\sum_{i=1}^{Z} \Theta_{i}\left(S_{A}\right)}{\sum_{i=1}^{Z} \Theta_{i}}
$$

where:

$\Theta_{i}\left(S_{A}\right)-i$-th realization of the time of remaining in the states of the modelled operating process, belonging to the availability states' sets $S_{A} \subset S=\{1,2, \ldots 10\}$,

$\Theta_{i}-i$-th realization of the time of remaining in the states of the modelled operating process $S=\{1,2, \ldots, 10\}$,

$Z=\mathrm{NOT} \cdot \mathrm{NZ}$ - number of occurences (changes of the model's states), for a determined number of technical objects;

- result determining the value of the average unit income

$$
C(\delta)=\frac{\sum_{i=1}^{Z} c_{i} \cdot \Theta_{i}}{\sum_{i=1}^{Z} \Theta_{i}}
$$

where:

$c_{i}-i$-th realization of the unit income concerning remaining in the operating process's modeling states $S=\{1,2, \ldots 10\}$.

\section{Exemplary results of the simulation model's researches}

The presented example has been developed based on the operating data obtained from the researches concerning the real operating system of the means of transport (municipal transport services' buses). In the studied transport system, there are used 160 municipal transport service buses, and the service and repair processes are conducted at the technical back-up facilities' stations and by the technical service units. A group of 33 vehicles aged from 2 to 4 years has been selected for research purposes. For the considered model of the means of transport operating process, there have been determined the values of the simulation model's input parameters, and also based on operating data, the values of the elements of the transition probability matrices $P$, possible decisions taken in decisive process's states (table 1), and the mean values of the duration times and the mean unit incomes generated in the process's states (table 2) have been determined. 


$$
P=\left|\begin{array}{cccccccccc}
0 & 1 & 0 & 0 & 0 & 0 & 0 & 0 & 0 & 0 \\
0 & 0 & 0.2320 & 0.0030 & 0 & 0 & 0.4988 & 0 & 0.2661 & 0 \\
0 & 0 & 0 & 0 & 0 & 1 & 0 & 0 & 0 & 0 \\
0 & 0 & 0 & 0 & 1 & 0 & 0 & 0 & 0 & 0 \\
0 & 0 & 0 & 0 & 0 & 1 & 0 & 0 & 0 & 0 \\
0 & 0.4702 & 0 & 0 & 0 & 0 & 0 & 0 & 0.5298 & 0 \\
0 & 0 & 0 & 0 & 0 & 0 & 0 & 0.0799 & 0.9201 & 0 \\
0 & 0 & 0 & 0 & 0 & 0 & 0 & 0 & 1 & 0 \\
0 & 0.2242 & 0 & 0 & 0 & 0 & 0 & 0 & 0 & 0.7758 \\
1 & 0 & 0 & 0 & 0 & 0 & 0 & 0 & 0 & 0
\end{array}\right|
$$

Table 1. Decisions at decision-making states of the analysed process.

\begin{tabular}{|c|c|c|}
\hline $\begin{array}{l}\text { Process } \\
\text { state }\end{array}$ & Decision "0" & Decision "1" \\
\hline 2 & $\begin{array}{l}\text { Route with "light" conditions for } \\
\text { completing the transport task }\end{array}$ & $\begin{array}{l}\text { Route with "heavy" conditions for } \\
\text { completing the transport task }\end{array}$ \\
\hline 5 & Post-collision intervention - "intensive" & Post-collision intervention - "normal" \\
\hline 6 & $\begin{array}{l}\text { Reconditioning following damage or } \\
\text { collision - extended scope }\end{array}$ & $\begin{array}{l}\text { Reconditioning following damage or } \\
\text { collision - basic scope }\end{array}$ \\
\hline 7 & Preventive diagnosis - extended scope & Preventive diagnosis - basic scope \\
\hline 8 & $\begin{array}{l}\text { Preventive reconditioning - extended } \\
\text { scope }\end{array}$ & Preventive reconditioning - basic scope \\
\hline 9 & Supplying - intensive & Supplying - normal \\
\hline 10 & Handling - intensive & Handling - normal \\
\hline
\end{tabular}

Table 2. Mean lengths of time and unit revenues at the states of the analyzed process depending on decision.

\begin{tabular}{|c|c|c|c|c|}
\hline $\begin{array}{c}\text { Process } \\
\text { state }\end{array}$ & $\Theta_{i}^{(0)}[\mathbf{h}]$ & $\Theta_{i}^{(1)}[\mathbf{h}]$ & $c_{i}^{(0)}[\mathbf{P L N} / \mathbf{h}]$ & $c_{i}^{(1)}[\mathbf{P L N} / \mathbf{h}]$ \\
\hline 1 & 5.659 & 5.659 & -6.77 & -6.77 \\
\hline 2 & 8.852 & 7.967 & 28.44 & 33.56 \\
\hline 3 & 0.514 & 0.514 & -69.08 & -69.08 \\
\hline 4 & 0.873 & 0.873 & -162.80 & -162.80 \\
\hline 5 & 1.712 & 2.372 & -304.63 & -234.11 \\
\hline 6 & 2.633 & 3.342 & -144.08 & -118.21 \\
\hline 7 & 0.441 & 0.677 & -82.71 & -54.98 \\
\hline 8 & 1.665 & 2.140 & -124.79 & -96.89 \\
\hline 9 & 0.096 & 0.152 & -23.09 & -17.46 \\
\hline 10 & 0.354 & 0.582 & -221.74 & -164.72 \\
\hline
\end{tabular}


Below there are presented the exemplary results of the simulation model's tests. The realized experiments consisted in 30 tuple simulation of the operating process for each considered strategy $\delta$. As a result of the simulation experiments' realization, there were obtained the sets of 30 values of the technical objects $A(\delta)$ availability and 30 values of the unit income $C(\delta)$. In the presented model, there have been distinguished the following technical objects availability states: 1,2 .

In figures 3 and 4 there are presented the exemplary results of simulation experiments for the selected strategies of the technical objects operating process's steering $\delta$, in such a manner, as the determined technical objects availability $A(\delta)$ be at least equal to the availability $A_{r}(\delta)$ required for correct realization of the transportation tasks (for $\left.A_{r}(\delta)=0.87,0.88,0.89,0.90\right)$. However, in the table 3 and in figures 5 and 6 there are presented the mean values, the values of standard deviation and the values of position statistics (minimum, 1 quartile, median, 3 quartile, maximum), determined for the considered characteristics of the technical objects performance assessment and for the selected strategies $\delta$.

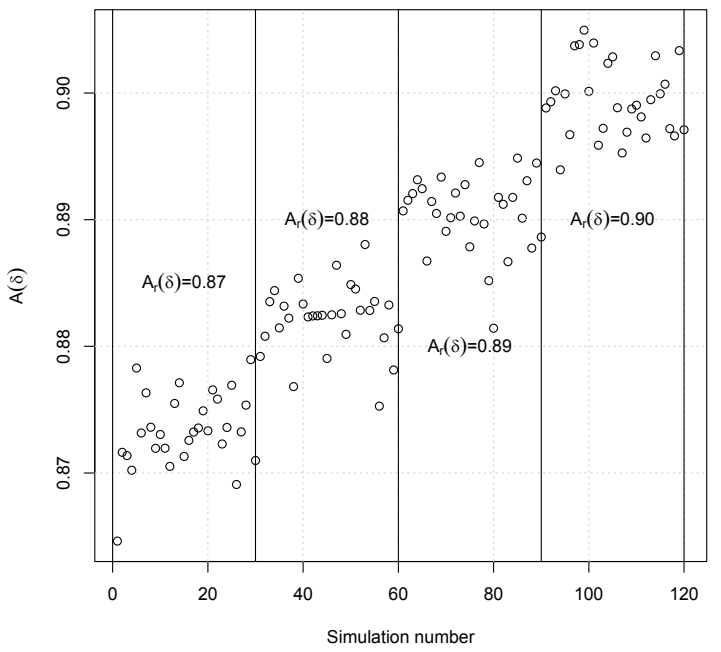

Fig. 3. Values of the technical objects availability determined based on the simulation experiments.

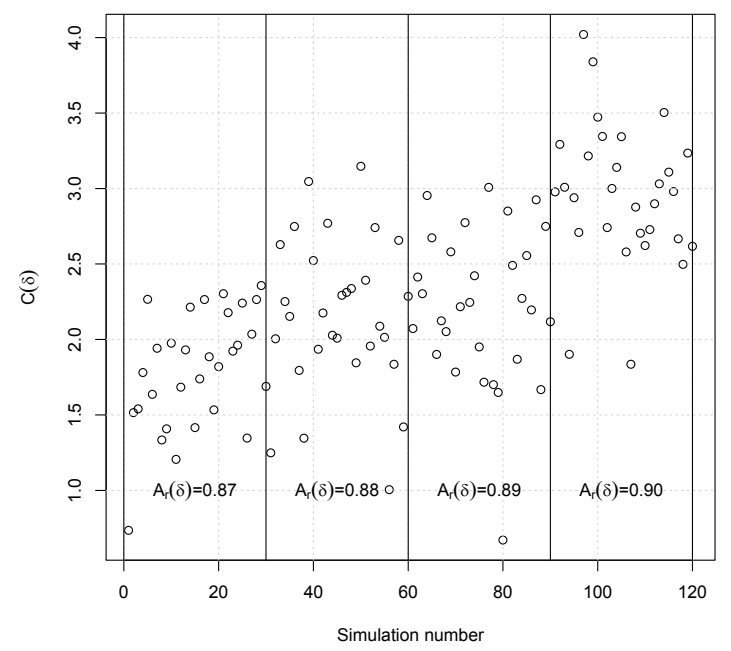

Fig. 4. Values of the unit income determined based on simulation experiments. 
Table 3. Values of statistics determined for the technical objects availability and unit incomes in $[\mathrm{PLN} / \mathrm{h}]$.

\begin{tabular}{|c|c|c|c|c|c|c|c|c|}
\hline & \multicolumn{2}{|c|}{$A_{r}(\boldsymbol{\delta})=\mathbf{0 . 8 7}$} & \multicolumn{2}{c|}{$A_{r}(\boldsymbol{\delta})=\mathbf{0 . 8 8}$} & \multicolumn{2}{c|}{$A_{\boldsymbol{r}}(\boldsymbol{\delta})=\mathbf{0 . 8 9}$} & \multicolumn{2}{|c|}{$A_{r}(\boldsymbol{\delta})=\mathbf{0 . 9 0}$} \\
\hline & $A(\delta)$ & $C(\delta)$ & $A(\delta)$ & $C(\delta)$ & $A(\delta)$ & $C(\delta)$ & $A(\delta)$ & $C(\delta)$ \\
\hline Medium & 0.8734 & 1.8034 & 0.8822 & 2.1660 & 0.8905 & 2.2302 & 0.8995 & 2.9610 \\
\hline $\begin{array}{c}\text { Standard } \\
\text { deviation }\end{array}$ & 0.0029 & 0.3886 & 0.0027 & 0.5071 & 0.0029 & 0.5038 & 0.0029 & 0.4687 \\
\hline Minimum & 0.8646 & 0.7353 & 0.8753 & 1.0050 & 0.8814 & 0.6714 & 0.8939 & 1.8360 \\
\hline 1 quartile & 0.8717 & 1.5352 & 0.8811 & 1.9410 & 0.8892 & 1.9131 & 0.9871 & 2.7050 \\
\hline Median & 0.8732 & 1.8525 & 0.8825 & 2.1640 & 0.8910 & 2.2310 & 0.8992 & 2.9790 \\
\hline 3 quartile & 0.8755 & 2.1422 & 0.8835 & 2.4900 & 0.8924 & 2.5742 & 0.9019 & 3.2300 \\
\hline Maximum & 0.8789 & 2.3575 & 0.8880 & 3.1470 & 0.8949 & 3.0080 & 0.9050 & 4.0200 \\
\hline
\end{tabular}

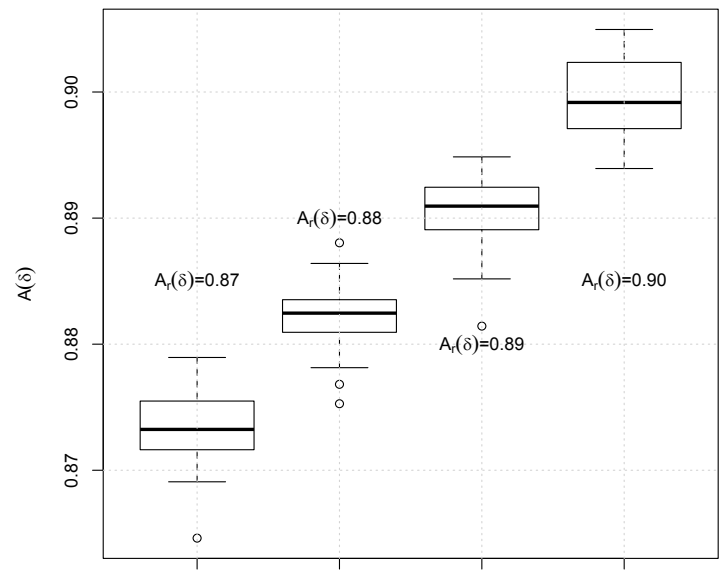

Required availability level

Fig. 5. Box-plot diagram of the technical objects availability value distribution.

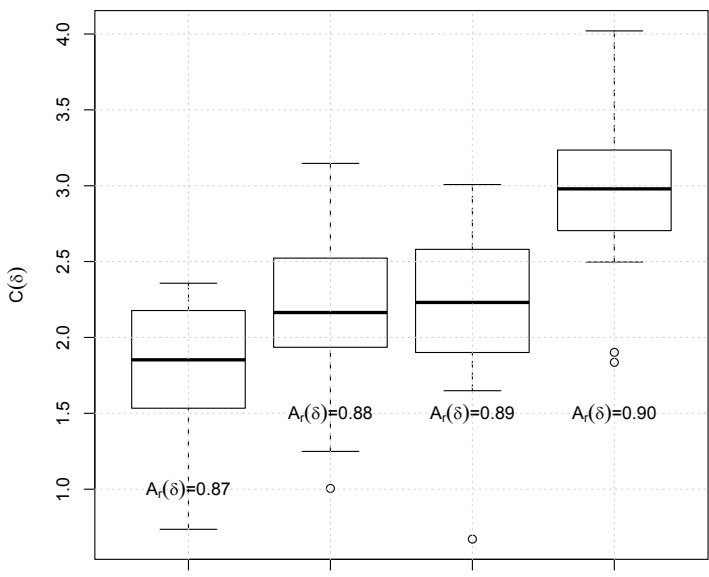

Required availability level

Fig. 6. Box-plot diagram of the unit income value's distribution. 


\section{Summary}

Based on the obtained results it may be noticed, that in the analysed operating system, the increase of the means of transport's availability results in the increase of the unit income's value. It is caused mainly by the fact, that the tested vehicles belonged to the group of the newest vehicles (age of the vehicles from 2 to 3 years), and thereby these were the vehicles in case of which there was no necessity to incur considerable costs for their treatment.

Analysing the obtained results it may be noticed, that within the scope of the considered value of the availability required $A_{r}(\delta)$ from 0.87 to 0.90 , together with the increase of availability, there occurs the increase of the mean unit income's value $C(\delta)$ respectively from the value 1.8034 to the value $2.9610[\mathrm{PLN} / \mathrm{h}]$. In the presented example it may be noted, that the availability increase from the level from 0.87 to 0.89 , results in a slight increase of the unit income's value respectively from 1.8034 to 2.2302 [PLN/h], that is on average the increase of the availability factor's value by 0.1 , results in the increase of the unit income by the value of 0.2134 [PLN/h].

Obtaining of the highest, analysed level of technical objects availability (for $\left.A_{r}(\delta)=0.90\right)$, results in the highest change of the unit income's value $\Delta C(\delta)=2.9610$ $2.2302=0.7308[\mathrm{PLN} / \mathrm{h}]$. Generally, low level of the unit income in the studies system of means of transport's operating results from the fact, that the studied system is the strategical enterprise owned the city, and its task it is not to generate profit but only ensuring an effective realization of the transportations tasks at a given area.

The simulation model presented in the article, is a partial result of the conducted studies, the purpose of which is to develop a complex method of the means of transport operating process's steering with the use of decisive semi-Markov processes and nondeterministic methods of optimum solutions' assigning (evolutionary algorithms).

\section{References}

1. F. Grabski, Semi-Markov processes: Applications in system reliability and maintenance. (Amsterdam, Elsevier, 2014)

2. F. Grabski, Analiza ryzyka $w$ decyzyjnych semi-markowskich modelach procesu eksploatacji. Risk analysis in decision-making semi-Markov models of operation process, (XXXVIII Zimowa Szkoła Niezawodności. $38^{\text {th }}$ Winter Reliability School, 2010, Szczyrk)

3. L. Knopik, K. Migawa, A. Wdzięczny, Profit optimalization in operation systems. (Polish Maritime Research, 2016)

4. V. G. Kulkarni, Modeling and analysis of stochastic systems (New York, Chapman \& Hall, 1995)

5. K. W. Lee, Stochastic models for random-request availability (IEEE Trans, Reliability, 2000)

6. P. Marbach, T. N. Tsitsiklis, Simulation based optimization of Markov reward process (IEEE Trans. Automat. Contr, 2001)

7. K. Migawa, A. Neubauer, Ł. Muślewski, B. Landowski, Method of availability control in the transport system using simulation model of operation and maintenance process (Journal of KONBiN, 2017)

8. K. Migawa, L. Knopik, A. Neubauer, D. Perczyński, Simulation of the model of technical object availability control (Engineering Mechanics, 2017, Czech Republic)

9. K. Migawa, L. Knopik, S. Wawrzyniak, Application of genetic algorithm to control the availability of technical systems (Engineering Mechanics, 2016, Czech Republic)

10. Ł. Muślewski, K. Migawa, L. Knopik, Control of technical objects operation quality with the use of simulation modeling PE (Risk, Reliability and Safety: Innovating Theory and Practice: Proceedings of ESREL, 2016) 
11. M. Szubartowski, K. Migawa, L. Knopik, M. Gniot, Simulation model of risk evaluation in transport system (Proceedings of $58^{\text {th }}$ ICMD, 2017, Prague, Czech Republic)

12. M. Woropay, K. Migawa, Markov model of the operational use process in an autonomous system. (Polish J. Environmental Studies, 2007)

13. R. Kostek, P. Aleksandrowicz, Simulation of car collision with an impact block (11th International Congress of Automotive and Transport Engineering, Pitesti, Romania, 2017)

14. R. Kostek, P. Aleksandrowicz, Simulation of the right-angle car collision based on identified parameters (11th International Congress of Automotive and Transport Engineering, Pitesti, Romania, 2017) 